\title{
A Parametric Investigation of Pattern Separation Processes in the Medial Temporal Lobe
}

\author{
Sarah E. Motley ${ }^{1,3}$ and C. Brock Kirwan ${ }^{2,3}$ \\ Departments of ${ }^{1}$ Physiology and Developmental Biology and ${ }^{2}$ Psychology and ${ }^{3}$ Neuroscience Center, Brigham Young University, Provo, Utah 84602
}

The hippocampus is thought to be involved in memory formation and consolidation, with computational models proposing the process of pattern separation as a means for encoding overlapping memories. Previous research has used semantically related targets and lures to investigate hippocampal responses to mnemonic interference. Here, we attempted to define the response function of the human hippocampus and its inputs during pattern separation by parametrically varying target-lure similarity in a continuous recognition task. We also investigated the effect of task demands (intentional vs incidental encoding) on pattern separation processes. We collected functional magnetic resonance imaging data while participants were shown a series of objects. In the intentional paradigm, participants identified objects as "new" (novel stimuli), "old" (exact repetitions), or "rotated" (previously seen objects that were subsequently rotated by varied degrees). In the incidental paradigm, participants were shown the same stimuli but identified objects as "toy" or "not toy." Activation in the hippocampus was best fit with a power function, consistent with predictions made by computational models of pattern separation processes in the hippocampus. The degree of pattern separation was driven by the information most relevant to the task: pattern separation was seen in the left hippocampus when semantic information was more important to the task and seen in the right hippocampus when spatial information was more important. We also present data illustrating that top-down processes modulate activity in the ventral visual processing stream.

\section{Introduction}

The hippocampus is involved in memory creation and consolidation (Squire et al., 2004), but its specific involvement is under current investigation. One hallmark of the hippocampus is its ability to store fast, non-overlapping representations of similar items or events and to retrieve these representations given a partial or degraded cue. Computational models term these processes pattern separation and pattern completion, respectively (Marr, 1971; Treves and Rolls, 1994; McClelland et al., 1995; O'Reilly and Rudy, 2001; Norman and O'Reilly, 2003; for a recent review, see Yassa and Stark 2011). Pattern separation orthogonalizes memory representations by magnifying small input changes, treating similar stimuli more like novel stimuli than repeated stimuli (Norman and O'Reilly, 2003). Pattern separation reduces interference, allowing efficient retrieval of specific memories without recalling memories with overlapping similarity (McClelland et al., 1995).

Sparse representations in the dentate gyrus subregion are thought to bias the hippocampus toward pattern separation (Treves and Rolls, 1994), whereas CA3 back-projections allow pattern completion (Amaral and Witter, 1989; Treves and Rolls,

\footnotetext{
Received Nov. 28, 2011; revised July 13, 2012; accepted July 20, 2012.

Author contributions: C.B.K. designed research; S.E.M. and C.B.K. performed research;S.E.M. and C.B.K. analyzed data; S.E.M. and C.B.K. wrote the paper.

This research was supported by a pilot grant from the Utah Brain Institute and by Brigham Young University's Mentoring Environment Grant. We thank the members of the University of Utah Imaging and Neurosciences Center for their assistance with fMRI data acquisition.

Correspondence should be addressed to C. Brock Kirwan, 1052 SWKT, Brigham Young University, Provo, UT 84602. E-mail: kirwan@byu.edu.

DOI:10.1523/JNEUROSCI.5920-11.2012

Copyright $\odot 2012$ the authors $\quad 0270-6474 / 12 / 3213076-09 \$ 15.00 / 0$
}

1994; Eichenbaum et al., 2007). The cortex can also pattern separate (Rotshtein et al., 2005), but computational models propose that the cortex requires a larger input change and/or more extensive training than the hippocampus to generate the same change in output (McClelland et al., 1995; O'Reilly and Rudy, 2001; Norman and O'Reilly, 2003).

Electrophysiology (Leutgeb et al., 2004, 2005; Wills et al., 2005; Leutgeb and Leutgeb, 2007), lesion (Lee and Kesner, 2004; Kesner, 2009), and immediate-early gene (Vazdarjanova and Guzowski, 2004; Kubik et al., 2007) rodent studies demonstrate hippocampal pattern separation. However, there is little human research of pattern separation, and methods have been modified for humans to test perceptually similar objects compared with the spatial contexts used with rodents.

Previous studies (Kirwan and Stark, 2007; Bakker et al., 2008) have used semantically related targets and lures to investigate hippocampal responses to mnemonic interference, without explicitly controlling target-lure similarity. Importantly, activity for high- and low-overlap lures was averaged together. Lacy et al. (2011) attempted to control target-lure similarity by using "low-similarity" or "high-similarity" lures. We used parametrically rotated objects to explicitly control target-lure similarity, hypothesizing that lures are not treated equally. We propose that pattern-separating regions will have large differences in functional magnetic resonance imaging (fMRI) activity in response to small changes in stimulus similarity, whereas pattern-completing regions will only have large differences when the similarity exceeds a threshold (Fig. 1).

Previous studies investigating intentional (Kirwan and Stark, 2007) and incidental (Bakker et al., 2008; Lacy et al., 2011) encoding have shown task-dependent hippocampal pattern sep- 
Pattern Separation

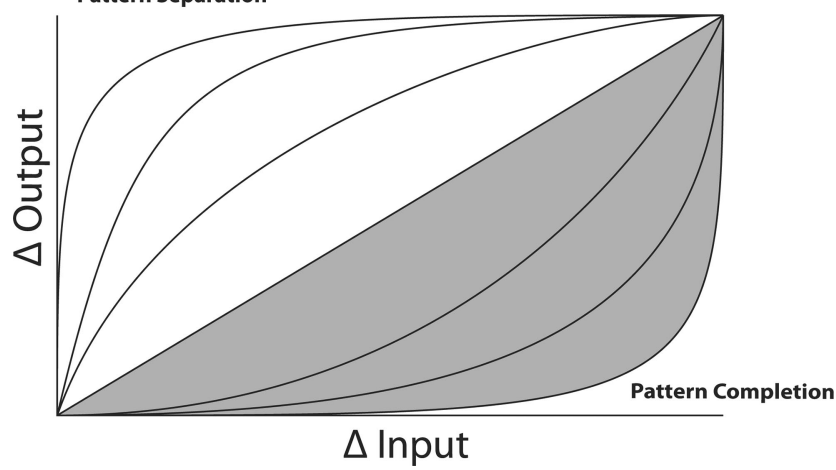

Figure 1. Graph of hypothetical activity curves for pattern separation and pattern completion, as predicted by computational models. Predictions are that regions performing pattern separation have a sharp distinction in activity for true repeats versus small rotations (i.e., a power function with a decreasing slope), whereas regions performing pattern completion have a more gradual response to the parametric rotations (i.e., a linear function or power function with increasing slope). Curves above the diagonal (area in white) represent pattern separation. Curves below the diagonal and including the diagonal (area in gray) represent pattern completion.
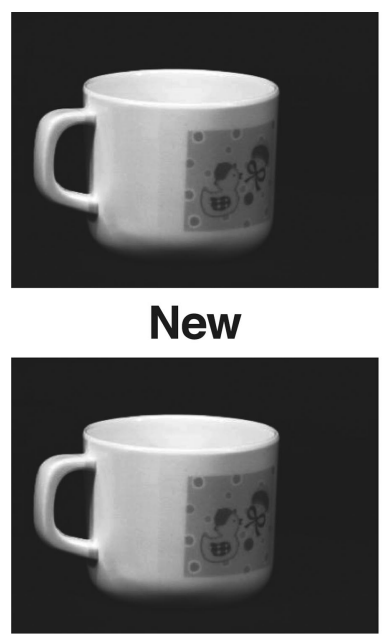

New

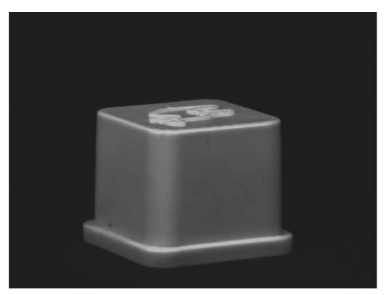

New

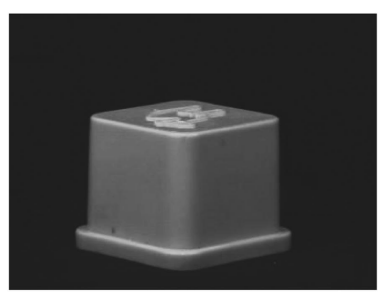

Rotated (15)

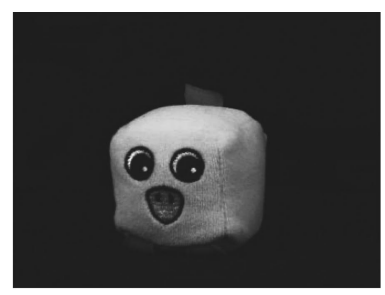

New

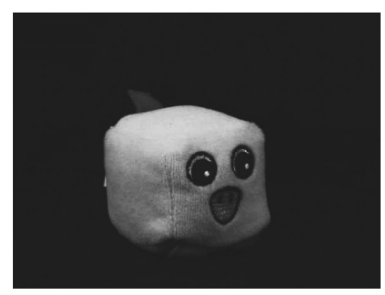

Rotated $\left(55^{\circ}\right)$

Figure 2. Examples of stimuli presented in the intentional task. During first presentation, all stimuli were classified as new. During second presentation, stimuli were classified as old or rotated depending on their orientation. Old stimuli were exact copies of the original image (i.e., rotated $0^{\circ}$ ). Rotated stimuli were rotated $15^{\circ}, 25^{\circ}, 35^{\circ}$, or $55^{\circ}$. Note that stimuli in the experiment were presented in full color.

aration activity. One study (Duncan et al., 2009) found a hippocampal match signal generated in an intentional task but a hippocampal mismatch signal generated in an incidental task. We proposed both an incidental and intentional design to investigate whether task demands modulate the response function within the hippocampus and its inputs (i.e., medial temporal lobe cortex and visual processing stream). We hypothesized that intentional encoding facilitates hippocampal pattern separation by sharpening the distinction between "old" and "similar" stimuli. Furthermore, we investigated where task-driven differences in hippocampal output were generated. We hypothesized that topdown processing of task demands facilitates pattern separation by sharpening ventral stream inputs into the hippocampus.

\section{Materials and Methods}

Participants. Thirty right-handed volunteers (15 in the intentional condition, eight females, mean age of 23.1 years, range of $18-27$ years; 15 in the incidental condition, seven females, mean age of 23.9 years, range of 18-29 years) gave written, informed consent before participation.

Materials. Stimuli consisted of images acquired from the Amsterdam Library of Images database (Geusebroek et al., 2005). The database contained photographs of 1000 single, small objects. Each object was photoraphed 72 times by rotating the object at $5^{\circ}$ intervals. Five hundred ninety-two objects were selected from the database for presentation in the continuous recognition task (see below). Objects were presented in color in the center of the screen against a white background. Response ptions were presented across the bottom of the screen in black letters.

Procedure: intentional condition. At the time of scanning, participants one at a time for $2.5 \mathrm{~s}$, and asked to identify each object as "new" (novel stimuli), "old" (exact repetitions), or "rotated" (previously seen objects shown from a different angle; Fig. 2). Pilot testing indicated that rotations of $15^{\circ}, 25^{\circ}, 35^{\circ}$, and $55^{\circ}$ were optimal for obtaining an appropriate range of performance. We defined this appropriate range of performance such that the percentage correct for each trial type was significantly different from all other trial types and that the percentage correct for the highest the was above $50 \%$. Each block of 198 trials included 10 objects that were repeated, 40 objects that were subsequently rotated (10 per angle of rotation), and 98 objects that were not repeated. Object orientation for the initial representation was randomly selected from the 72 possible orientations to prevent answer bias for the subsequent rotated trials. Images that were subsequently repeated were treated as the $0^{\circ}$ in the first presentation, regardless of initial object orientation. Presenting and randomly repeating stimuli from the same category accomplished interstimulus jitter. Whereas task stimuli were presented every $2.5 \mathrm{~s}$, stimuli of a given trial type (e.g., objects rotated $15^{\circ}$ ) were presented with a random jitter. Repeat images were shown after a mean lag of 20.9 trials (range, 11-51). Rotated images were shown after a mean lag of 19.8 trials (range, 10-41).

The direction of rotation (clockwise or counterclockwise) was randomly selected. The same images were used for all participants but were pseudorandomized and were repeated only within the block. Block order was randomized across participants. Round or cylindrical stimuli were not included as old or rotated objects because of their symmetry but were included as new objects. Participants responded using an MR-compatible button box with buttons corresponding to the response options. Participants were instructed to respond while the image was still present on the screen.

Procedure: incidental condition. The same stimuli and stimulus presentation order were used in the incidental condition as in the intentional condition. At the time of scanning, participants were shown a series of images and asked to identify the objects as "toy" or "not toy." Participants were told to consider an object a toy if they could imagine a child playing with it. The percentage of toy versus non-toy objects cannot be calculated, because this is a subjective measure dependent on participant response. The participants' classifications were not used in the analysis, but these instructions were given to encourage participants to attend to the stimuli in an attempt to mimic the time spent attending to the objects in the intentional paradigm. Of the items classified by participants, $37 \%$ of objects were identified as toy (range, $30-49 \%$ ) and $63 \%$ of objects as not a toy (range, $51-70 \%$ ).

fMRI imaging. Imaging was performed on a $3 \mathrm{~T}$ Siemens scanner at the Imaging and Neurosciences Center at the University of Utah. Functional images were acquired using a gradient-echo, echo-planar, T2*-weighted pulse sequence (TR, $2500 \mathrm{~ms} ; 198 \mathrm{TRs} / \mathrm{run}$; TE, $30 \mathrm{~ms}$; flip angle, $75^{\circ}$; matrix size, $64 \times 64$; field of view, $22 \mathrm{~cm}$ ). The first four TRs acquired were discarded to allow for T1 equilibration. Forty-two oblique coronal 
slices (slice thickness, $3.3 \mathrm{~mm}$ ) were acquired parallel with the corpus callosum and covering the whole brain. Slices were interleaved and collected without a skip.

Structural MRI images were acquired using a T1-weighted MP-RAGE sequence $\left(165 \times 220 \mathrm{~mm}\right.$ field of view; flip angle, $12^{\circ}$; TE, $2.58 \mathrm{~ms} ; 128$ slices; $1 \mathrm{~mm}$ slice thickness; matrix size, $144 \times 192$; voxel size, $1.46 \times$ $1.45 \times 1 \mathrm{~mm})$.

fMRI data analysis. fMRI data were analyzed using the AFNI suite of programs (Cox, 1996). Functional data were coregistered in three dimensions to the whole-brain anatomical data, slice-time corrected, and coregistered to reduce effects of head motion. Large motion events, defined as TRs in which there was $>0.3^{\circ}$ of rotation or $0.6 \mathrm{~mm}$ of translation in any direction, were excluded from the deconvolution analysis by censoring, which removed said time points from the data (mean of 0.4 events per participant). We also excluded the TR immediately before and after the motion-contaminated TR.

Seven behavioral vectors were created that coded each study trial according to type (new, first, repeat, rotated $15^{\circ}$, rotated $25^{\circ}$, rotated $35^{\circ}$, rotated $55^{\circ}$ ). Trials in which there was no response or multiple responses were modeled but then excluded from additional analysis (intentional mean, 20.4 per participant; incidental mean, 32.8 trials per participant). The behavioral vectors and six vectors that coded for motion (three for translations and three for rotations) were used in the deconvolution analysis of the fMRI time series data in which a canonical hemodynamic response was convolved with the behavioral vectors. The resultant fit coefficients ( $\beta$ coefficients) represent activity versus baseline in each voxel across the time series for a given trial type. Note that the stimuli in the response category new were further subdivided into first and new, in which firsts had a subsequent second presentation and new were never repeated. Baseline consisted of new stimuli that were never repeated. The following fMRI contrasts of interest were all made within active task conditions and not relative to baseline.

Initial spatial normalization was accomplished using each participant's structural MRI scan to transform the data to the atlas of Talairach and Tournoux (1988). Additional spatial normalization was performed using the Advanced Normalization Tools (ANTs) (Avants et al., 2008; Klein et al., 2009; Yassa et al., 2010; Lacy et al., 2011), which uses diffeomorphic mapping to calculate a transformation from an individual participant to a model based on the grayscale structural MRI scan. Statistical maps also were transformed to Talairach space and then resampled to 3 $\mathrm{mm}^{3}$ and smoothed using a Gaussian filter $(5 \mathrm{~mm}$ full-width at halfmaximum) before being aligned to the template with ANTs.

After individual deconvolution analysis, individual participant parameter estimate maps were entered into group-level analyses and thresholded at a voxelwise $p$ value of $<0.03$. A cluster correction technique was used to correct for multiple comparisons, and Monte Carlo simulations (AFNI ClustSim program) were used to determine how large a cluster of voxels was needed to be statistically meaningful $(p<0.05)$ (Forman et al., 1995; Xiong et al., 1995) for the entire brain. The minimum cluster spatial extent threshold was determined to be 55 voxels using the AFNI program 3dClustSim, which performed 20,000 iterations of Monte Carlo simulations on an anatomical mask dataset with 57,550 voxels.

\section{Results}

\section{Behavioral performance}

Figure 3 shows the mean number of trials for each condition according to the behavioral response for the intentional version of the task. Percentage correct was calculated as the proportion of trials for which participants correctly identified the object (i.e., the number of times the participant identified an old object as old divided by the total number of old stimuli). Participants were highly accurate at identifying novel and repeat stimuli $(89,87$, and $75 \%$ for the new, first, and repeat presentations, respectively). Accuracy for correctly identifying second-presentation stimuli was positively correlated with increasing the angle of rotation $\left(22,35,50\right.$, and $56 \%$ for stimuli rotated $15^{\circ}, 25^{\circ}, 35^{\circ}$, and $55^{\circ}$, respectively; $\left.r=0.63 ; p<0.0001\right)$. Because successful pat-

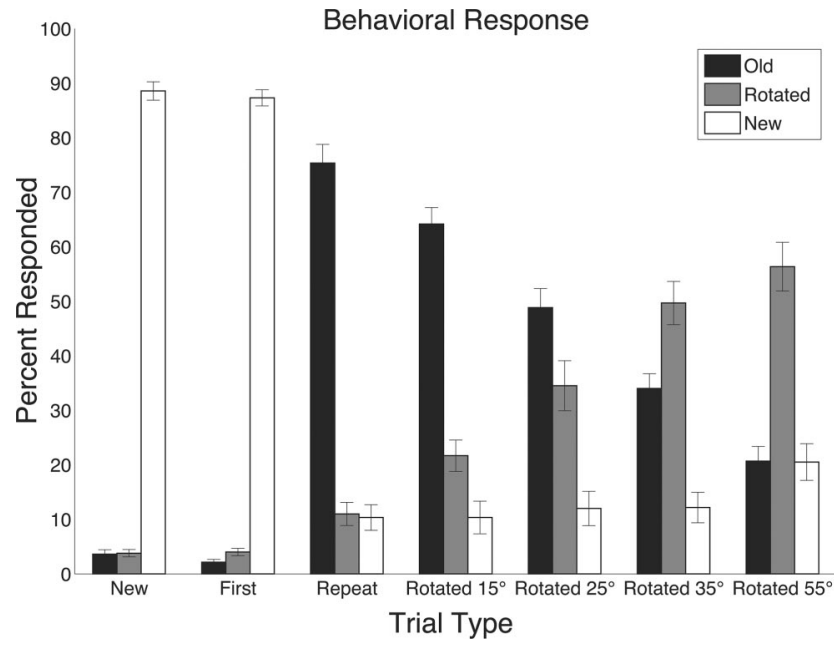

Figure 3. Behavioral performance during the intentional encoding paradigm. Response rates were taken out of the total number of trials of a given type (e.g., repeats) for which there was a behavioral response.

tern separation is indicated behaviorally by correctly discriminating similar objects, here pattern separation accuracy increased as the angle of rotation increased (i.e., as the objects became less similar).

\section{fMRI analyses}

Our primary purpose was to examine the response function of the medial temporal lobe (MTL) to parametric manipulations of stimulus similarity. To define memory-sensitive regions, we first looked for areas that showed an old/new effect by performing a voxelwise $t$ test comparing the repeat condition (old stimuli) and the baseline condition (new stimuli that were never repeated). Previous research with similar stimuli and procedures (Bakker et al., 2008) has shown regions performing both pattern separation and pattern completion that demonstrate higher activity for new than for old stimuli. Accordingly, we used this contrast as a mask to isolate areas that are sensitive to item repetition and then interrogate them for evidence of either pattern separation or pattern completion. To perform this contrast, data were collapsed across the incidental and intentional conditions, allowing us to use the same $t$ test to isolate voxels that exhibited a difference between new and old stimuli in either the incidental or the intentional task. A two-tailed $t$ test revealed two regions of interest (ROIs) in the MTL that exhibited an old/new effect (i.e., new > old). There were no ROIs in the MTL that exhibited an effect of old $>$ new. For this analysis, functional ROIs were defined by setting a voxelwise threshold at $p=0.03$ and a spatial extent threshold of a minimum ROI volume of $1485 \mathrm{~mm}^{3}$ (55 voxels) with a connectivity radius of $3.1 \mathrm{~mm}$ (overall $p<0.05$; see Materials and Methods). The resulting large functional ROIs spanned both hippocampus and the adjacent MTL cortex. Accordingly, these large ROIs were then masked to exclude nonMTL voxels and to segregate the functional MTL ROIs into their respective anatomical components using the MTL anatomical model from the cross-participant alignment procedure. Note that the resulting anatomically segmented ROIs fall below the spatial extent threshold described above. Figure 4 shows the four ROIs in the MTL revealed by masking: one in the left hippocampus body and tail, one in the right hippocampus body and tail, one in the left parahippocampal cortex, and one in the right parahippocampal cortex (Table 1). It is of note that the behav- 
A
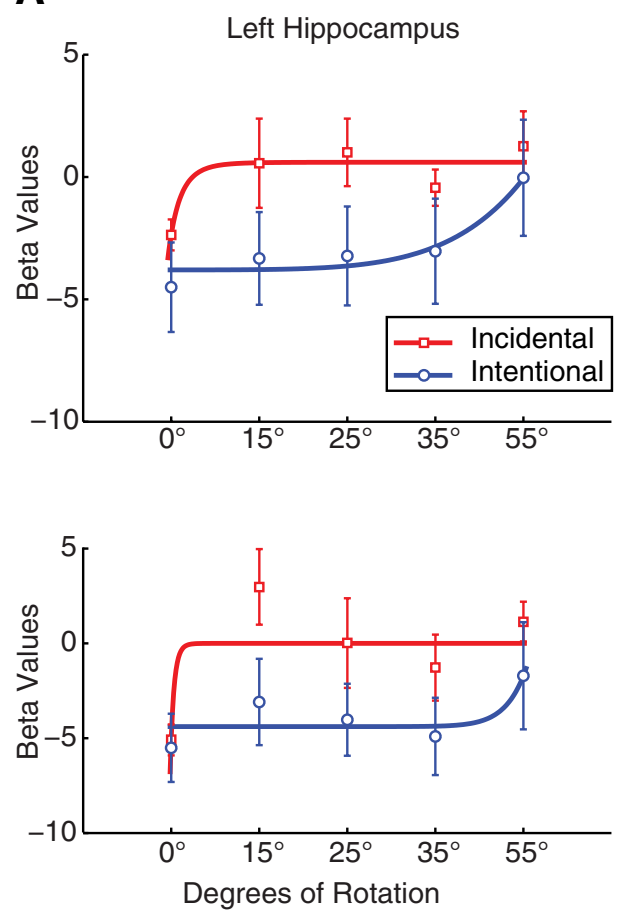

B
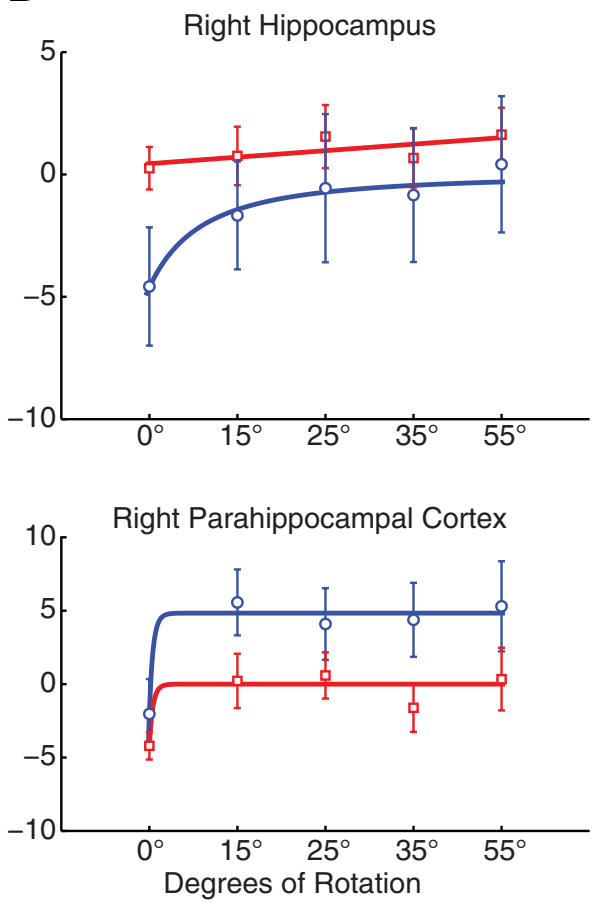
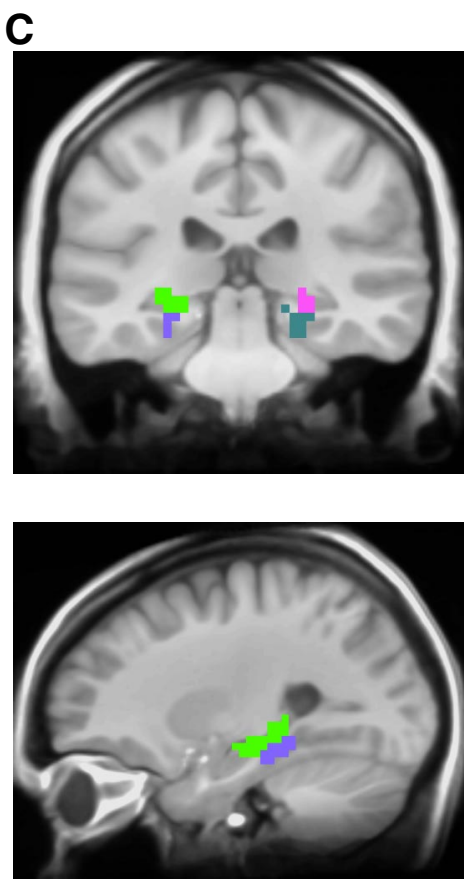

Figure 4. $\quad \boldsymbol{A}, \boldsymbol{B}$, Activity during the incidental (red) and intentional (blue) encoding conditions in the left $(\boldsymbol{A})$ and right $(\boldsymbol{B})$ hippocampus and parahippocampal cortex. Brackets show \pm SEM. Mean values for the first presentation are represented by the 0 line on the $y$-axis. Left Hippocampus, Intentional, adjusted $R^{2}=0.84$; incidental, adjusted $r=0.62$. Left Parahippocampal Cortex, Intentional, adjusted $R^{2}=0.26$; incidental, adjusted $r=0.57$. Right Hippocampus, Intentional, adjusted $R^{2}=0.93$; incidental, adjusted $r=0.33$. Right Parahippocampal Cortex, Intentional, adjusted $R^{2}=0.92$; incidental, adjusted $r=0.75$. C, Coronal (top) and sagittal (bottom) slices display the hippocampal and parahippocampal cortex R0ls: left hippocampus, lime green; left parahippocampal cortex, purple; right hippocampus, pink; right parahippocampal cortex, teal.

Table 1. ROIs isolated in the intentional versus incidental and first versus repeat $t$ test contrasts

\begin{tabular}{|c|c|c|c|c|c|}
\hline Contrast & Region & $x$ & $y$ & $z$ & $\mu l$ \\
\hline \multirow{4}{*}{$\begin{array}{l}\text { Intentional versus incidental ( } t \text { test) } \\
\text { (intentional }>\text { incidental) }\end{array}$} & L Ventral & 34 & 80 & -2 & 33,831 \\
\hline & R Ventral & -28 & 88 & 0 & 24,786 \\
\hline & L MTL & 37 & 12 & -25 & 3861 \\
\hline & R MTL & -29 & 26 & -21 & 4401 \\
\hline \multirow{4}{*}{$\begin{array}{l}\text { First versus repeat ( } t \text { test) (first }> \\
\text { repeat) }\end{array}$} & LH & 26 & 26 & -6 & 1566 \\
\hline & RH & -23 & 29 & -4 & 270 \\
\hline & LPHC & 23 & 32 & -10 & 891 \\
\hline & RPHC & -21 & 33 & -10 & 999 \\
\hline \multirow{3}{*}{$\begin{array}{l}\text { Intentional versus incidental ( } t \text { test) } \\
\text { default network (intentional }< \\
\text { incidental) }\end{array}$} & $\begin{array}{l}\text { Mid, R Par, L DLFr, L VLFr, } \\
\text { R VLFr }\end{array}$ & -13 & 12 & 22 & 199,503 \\
\hline & L Par, L LTL & 54 & 47 & 16 & 22,896 \\
\hline & LDLFr & 45 & -15 & 4 & 12,256 \\
\hline
\end{tabular}

L, Left; Ventral, ventral stream; $R$, right; $H$, hippocampus; $P H C$, parahippocampal cortex; Mid, midline; Par, parietal cortex; DLFr, dorsolateral frontal cortex; VLFr, ventrolateral frontal cortex; LTL, lateral temporal lobe.

ioral data and the functional data diverge from one another (e.g., although the behavioral performance improves in a linear manner, the fMRI activation is not necessarily linear). This is consistent with previous research (Daselaar et al., 2006; Kirwan et al., 2009), which has shown that the hippocampus activity is not entirely coordinated with the participant's behavioral response.

We next looked specifically within the MTL ROIs to define the response function to parametric manipulations of stimulus similarity. Figure 4 shows the mean activity within each of the ROIs (represented by $\beta$ values) for the trials rotated $0^{\circ}$ (repeats), $15^{\circ}$, $25^{\circ}, 35^{\circ}$, and $55^{\circ}$. Because first presentations were used for the baseline, the 0 line on the $y$-axis represents the mean activity of this condition. We looked at activity in these regions across stimulus type for evidence of either pattern separation or pattern completion. Before our investigations, we proposed model curves for pattern separation and pattern completion (Fig. 1). Pattern separation was defined as a power function with decreasing slope based on computational predictions that a sharp distinction in activity exists between old and similar stimuli (i.e., a distinction between repeat and small-rotation images). Pattern completion was defined as either a linear function or a power function with increasing slope based on computational predictions that a more gradual distinction in activity exists between old and similar stimuli. As the slope of the activity curve becomes less steep, both the pattern separation and the pattern completion curves approach the linear function shown in the middle of Figure 1 . The regions in the MTL defined in this contrast are sensitive to stimulus repetition as they were defined by the new $>$ old contrast. Thus, memory-sensitive regions can be thought of as supporting one of three judgments, recognizing something as old, similar, or novel. The output from those regions distinctly reflects one of the three judgments. Because the level of activity does not mirror that of new or similar stimuli, we have defined a linear function as the limit of pattern completion. Accordingly, we fit a linear and a power function to the activity for trials with rotations of $0^{\circ}$ (repeats), $15^{\circ}, 25^{\circ}, 35^{\circ}$, and $55^{\circ}$ (Table 2). For this and subsequent analyses, areas were defined as performing pattern separation if the best-fit curve was a power function with decreasing slope (i.e., a marked difference in activity between the repeat and small-rotation conditions was observed). Areas were defined as performing pattern completion if the best-fit curve was either linear (i.e., activity changed proportional to the change in angle of rotation) or a power function with increasing slope (i.e., activity for the small-rotation conditions was similar to the repeat condition, but a marked difference was observed in activity of 
Table 2. Curves fit to the hippocampal and parahippocampal cortex ROIs

\begin{tabular}{|c|c|c|c|c|c|c|c|c|}
\hline ROI & Condition & Fit type & Model & Adjusted $R^{2}$ & $R^{2}$ & RMSE & SSE & DFE \\
\hline \multirow[t]{4}{*}{ LHy } & \multirow[t]{2}{*}{ Incidental } & \multirow[t]{2}{*}{ Linear power } & $f(x)=0.6249 x-1.87$ & 0.27 & 0.45 & 1.26 & 4.80 & 3 \\
\hline & & & $f(x)=-2.969 \times x^{\wedge}(-7.304)+0.6024$ & 0.62 & 0.82 & 0.92 & 1.67 & 2 \\
\hline & \multirow[t]{2}{*}{ Intentional } & \multirow[t]{2}{*}{ Linear power } & $f(x)=0.9237 x-5.596$ & 0.69 & 0.77 & 0.92 & 2.54 & 3 \\
\hline & & & $f(x)=0.0001894 \times x^{\wedge}(6.145)-3.797$ & 0.84 & 0.92 & 0.67 & 0.91 & 2 \\
\hline \multirow[t]{4}{*}{ L Phy } & \multirow[t]{2}{*}{ Incidental } & \multirow[t]{2}{*}{ Linear power } & $f(x)=0.8183 x-2.898$ & -0.09 & 0.18 & 3.15 & 29.81 & 3 \\
\hline & & & $f(x)=-5.075 \times x^{\wedge}(-23.96)$ & 0.57 & 0.68 & 1.98 & 11.73 & 3 \\
\hline & \multirow[t]{2}{*}{ Intentional } & \multirow[t]{2}{*}{ Linear power } & $f(x)=0.5788 x-5.584$ & 0.16 & 0.37 & 1.38 & 5.71 & 3 \\
\hline & & & $f(x)=1.488 \mathrm{e}-15 \times x^{\wedge}(21.82)-4.387$ & 0.26 & 0.63 & 1.30 & 3.36 & 2 \\
\hline \multirow[t]{4}{*}{ R Hy } & \multirow[t]{2}{*}{ Incidental } & \multirow[t]{2}{*}{ Linear power } & $f(x)=0.2658 x+0.1747$ & 0.33 & 0.50 & 0.49 & 0.71 & 3 \\
\hline & & & $f(x)=-1.807 \times x^{\wedge}(-0.5949)+2.058$ & 0.12 & 0.56 & 0.56 & 0.62 & 2 \\
\hline & \multirow[t]{2}{*}{ Intentional } & \multirow[t]{2}{*}{ Linear power } & $f(x)=1.082 x-4.695$ & 0.75 & 0.81 & 0.96 & 2.76 & 3 \\
\hline & & & $f(x)=-4.617 \times x^{\wedge}(-1.687)$ & 0.93 & 0.95 & 0.50 & 0.76 & 3 \\
\hline \multirow[t]{4}{*}{ R Phy } & \multirow[t]{2}{*}{ Incidental } & \multirow[t]{2}{*}{ Linear power } & $f(x)=0.7257 x-3.111$ & 0.09 & 0.32 & 1.93 & 11.16 & 3 \\
\hline & & & $f(x)=-4.204 \times x^{\wedge}(-25.03)$ & 0.75 & 0.81 & 1.02 & 3.11 & 3 \\
\hline & \multirow[t]{2}{*}{ Intentional } & \multirow[t]{2}{*}{ Linear power } & $f(x)=1.347 x-0.5775$ & 0.28 & 0.46 & 2.65 & 21.06 & 3 \\
\hline & & & $f(x)=-6.864 \times x^{\wedge}(-24.11)+4.835$ & 0.92 & 0.96 & 0.87 & 1.51 & 2 \\
\hline
\end{tabular}

L, Left; H, hippocampus; R, right; PHC, parahippocampal cortex; Linear, linear fit; Power, power fit; RMSE, root mean squared error; SSE, sum of squares error; DFE, degrees of freedom error.

large-rotation conditions.) The best fit was defined as having the highest adjusted $R^{2}$. The best-fit curve gives a qualitative interpretation of the function of the ROI in a given task condition. The adjusted $R^{2}$ value gives a quantitative measure of the fit of the curve, allowing not only a within-ROI comparison of the ROI function during the two task conditions but also a between-ROI comparison of the ROI in a given condition against any other ROI in either condition. The adjusted $R^{2}$ value also corrects for any differences in degrees of freedom when comparing the linear and power fits for a given dataset. It does this by dividing the $R^{2}$ obtained from the curve-fitting procedure by the relevant degrees of freedom.

Graphs of the activity were generated in MATLAB version 7.9 (MathWorks) using all five data points and were fit with either a linear or a power function using the curve-fitting toolbox of MATLAB. Curve fitting was done on group-level data to minimize noise from intersubject variability. Pilot testing indicated a linear increase in discriminability with $15^{\circ}, 25^{\circ}, 35^{\circ}$, and $55^{\circ}$ of rotation. Thus, levels of rotation, and not exact degrees, were chosen for $x$ values in the curve fitting, as reflected in Figures 4 and 5. The general formula for the power function was $f(x)=a \times$ $x^{b}+c$. The general formula for the linear function was $f(x)=a \times$ $x+b$. Curve fitting was accomplished using nonlinear leastsquares regression using the trust-region change algorithm. The toolbox was allowed to calculate the optimized start points for least-squares regression based on the mean activation within each ROI. It is likely that the curve fitting was mostly driven by the difference in activity between the $0^{\circ}$ rotation point and the $15^{\circ}$ rotation point, demonstrating that the response to rotated stimuli in these ROIs is more similar to the response to new stimuli than to old stimuli. This pattern of response is consistent with the hippocampus establishing a new representation for stimuli that only slightly differ from previous stimuli (i.e., pattern separation).

We fit separate curves to both the intentional and the incidental data in the two hippocampal ROIs (Table 2). In the incidental condition, the left hippocampus was better fit with a power function with decreasing slope (adjusted $R^{2}=0.62$ and 0.27 for the power and linear fits, respectively). The right hippocampus was better fit with a linear function (adjusted $R^{2}=0.33$ and 0.12 for the linear and power fits, respectively). In the intentional condition, the left hippocampus was better fit with a power function with increasing slope (adjusted $R^{2}=0.84$ and 0.69 for the power and linear fits, respectively). The right hippocampus was better fit with a power function with decreasing slope (adjusted $R^{2}=0.93$ and 0.75 for the power and linear fits, respectively). These data support the predictions of computational models of hippocampal function in that the response function in the hippocampus is consistent with pattern separation as determined by task demands.

Additionally, within the parahippocampal cortex ROIs (Fig. 4), we fit a linear and a power function to the activity from both the intentional and incidental task conditions (Table 2). In the incidental condition, the left parahippocampal cortex was better fit with a power function with decreasing slope (adjusted $R^{2}=$ 0.57 and -0.09 for the power and linear fits, respectively). The right parahippocampal cortex was better fit with a power function with decreasing slope (adjusted $R^{2}=0.75$ and 0.09 for the power and linear fits, respectively). In the intentional condition, the left parahippocampal cortex was better fit with a power function with increasing slope (adjusted $R^{2}=0.29$ and 0.16 for the power and linear fits, respectively). The right parahippocampal cortex was better fit with a power function with decreasing slope (adjusted $R^{2}=0.92$ and 0.28 for the power and linear fits, respectively). These data suggest a role of the parahippocampal cortex in signaling the hippocampus in pattern separation but that this role is modulated by task demands.

Last, we investigated the top-down processes modulating the effects of task demands via the inputs into the hippocampus. We first looked for areas that showed differential activation in the two task conditions by performing a voxelwise $t$ test comparing the intentional and the incidental encoding conditions. Because of widespread activation, for this analysis, functional ROIs were defined by setting a voxelwise threshold at $p=0.03$ and a spatial extent threshold of a minimum ROI volume of $2970 \mathrm{~mm}^{3}(110$ voxels) with a connectivity radius of $3.1 \mathrm{~mm}$ (overall $p<0.0001)$. Figure 5 shows the four ROIs revealed in this contrast in which activity was greater for the intentional condition than the incidental condition: one in the left ventral visual stream, one in the right ventral visual stream, one in the left MTL, and one in the right MTL (Table 1). Figure 5 shows the mean activity within each of the ROIs (represented by $\beta$ values) for the trials with objects rotated $0^{\circ}$ (repeats), $15^{\circ}, 25^{\circ}, 35^{\circ}$, and $55^{\circ}$. To classify this activity as consistent with either pattern separation or pattern completion, we fit a linear and a power function to the activity of all four ROIs in both the incidental and intentional conditions 
A

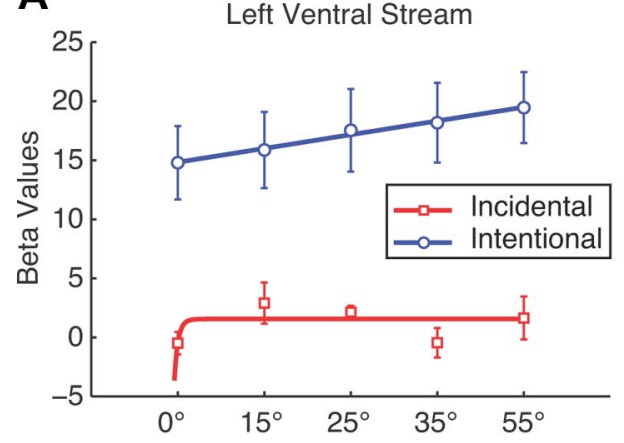

Left Medial Temporal Lobe

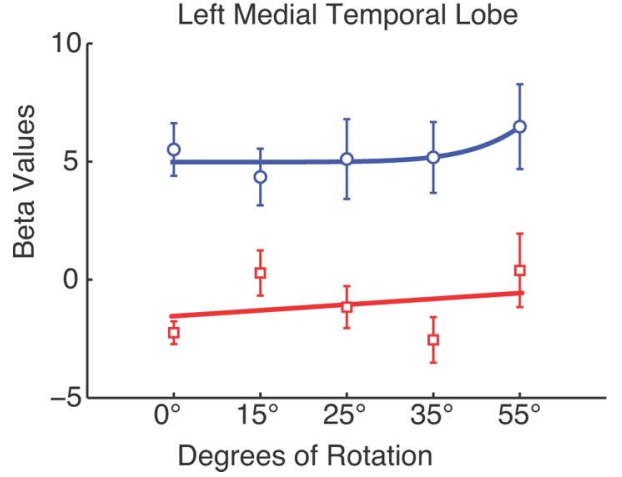

B

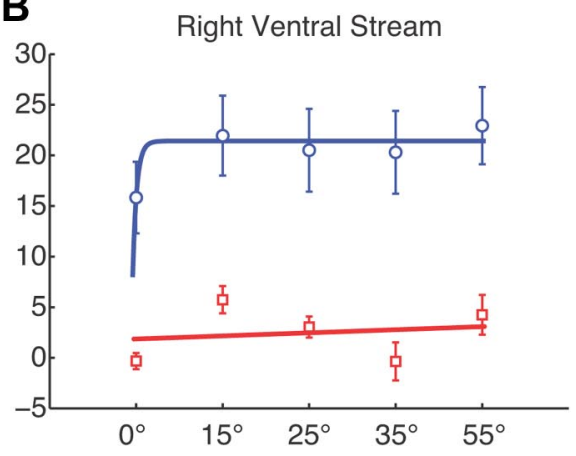

Right Medial Temporal Lobe

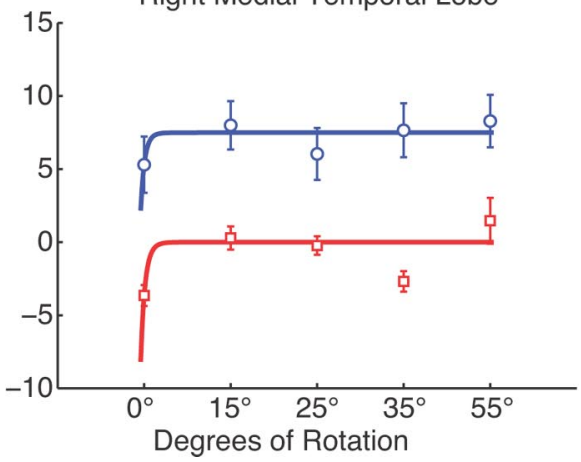

C
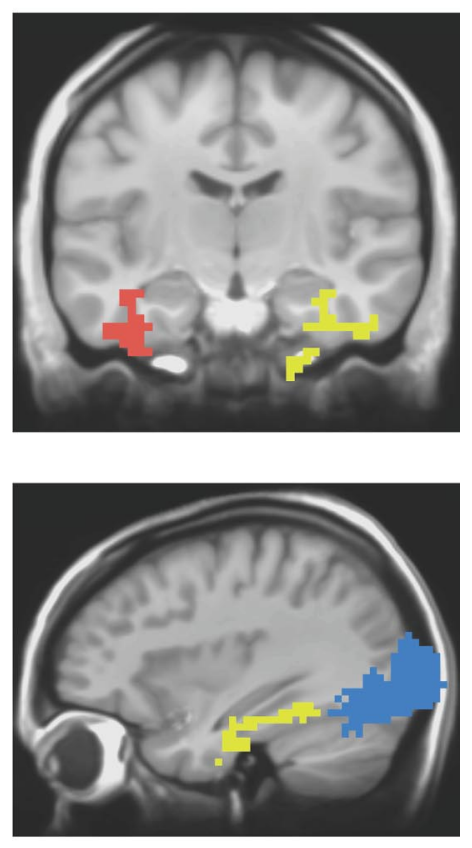

Figure 5. $\quad \boldsymbol{A}, \boldsymbol{B}$, Activity during the incidental (red) and intentional (blue) encoding conditions in the left $(\boldsymbol{A})$ and right ( $\boldsymbol{B})$ ventral streams and MTL. Brackets show \pm SEM. Mean values for the first presentation are represented by the 0 line on the $y$-axis. Left Ventral Stream, Intentional, adjusted $R^{2}=0.98$; incidental, adjusted $r=-0.30$. Left Medial Temporal Lobe, Intentional, adjusted $R^{2}=0.42$; incidental, adjusted $r=-0.23$. Right Ventral Stream, Intentional, adjusted $R^{2}=0.69$; incidental, adjusted $r=-0.30$. Right Medial Temporal Lobe, Intentional, adjusted $R^{2}=0.28$; incidental, adjusted $r=0.30$. C, Coronal (top) and sagittal (bottom) slices display the ventral stream and MTL ROls: left ventral stream, not pictured; left MTL, red; right ventral stream, Blue; right MTL, yellow.

Table 3. Curves fit to the ventral stream and MTL ROls

\begin{tabular}{|c|c|c|c|c|c|c|c|c|}
\hline ROI & Condition & Fit type & Model & Adjusted $R^{2}$ & $R^{2}$ & RMSE & SSE & DFE \\
\hline \multirow[t]{4}{*}{ L MTL } & \multirow[t]{2}{*}{ Incidental } & \multirow[t]{2}{*}{ Linear power } & $f(x)=0.244 x-1.787$ & -0.23 & 0.08 & 1.52 & 6.96 & 3 \\
\hline & & & $f(x)=-1.484 \times x^{\wedge}(-24.02)-0.7579$ & -0.53 & 0.23 & 1.70 & 5.79 & 2 \\
\hline & \multirow[t]{2}{*}{ Intentional } & \multirow[t]{2}{*}{ Linear power } & $f(x)=0.2758 x+4.498$ & 0.09 & 0.32 & 0.74 & 1.63 & 3 \\
\hline & & & $f(x)=1.101 e-06 \times x^{\wedge}(8.775)+4.8$ & 0.42 & 0.71 & 0.59 & 0.70 & 2 \\
\hline \multirow[t]{4}{*}{ L Ventral } & \multirow[t]{2}{*}{ Incidental } & \multirow[t]{2}{*}{ Linear power } & $f(x)=0.09061 x+0.8777$ & -0.32 & 0.01 & 1.78 & 9.47 & 3 \\
\hline & & & $f(x)=-2.047 \times x^{\wedge}(-22.95)+1.559$ & -0.30 & 0.35 & 1.76 & 6.20 & 2 \\
\hline & \multirow[t]{2}{*}{ Intentional } & \multirow[t]{2}{*}{ Linear power } & $f(x)=1.166 x+13.67$ & 0.98 & 0.99 & 0.25 & 0.18 & 3 \\
\hline & & & $f(x)=14.47 \times x^{\wedge}(0.1734)$ & 0.95 & 0.97 & 0.40 & 0.47 & 3 \\
\hline \multirow[t]{4}{*}{ RMTL } & \multirow[t]{2}{*}{ Incidental } & \multirow[t]{2}{*}{ Linear power } & $f(x)=0.726 x-3.141$ & 0.05 & 0.29 & 2.07 & 12.89 & 3 \\
\hline & & & $f(x)=-3.65 \times x^{\wedge}(-19.79)$ & 0.30 & 0.48 & 1.78 & 9.48 & 3 \\
\hline & \multirow[t]{2}{*}{ Intentional } & \multirow[t]{2}{*}{ Linear power } & $f(x)=0.5622 x+5.366$ & 0.12 & 0.56 & 1.23 & 3.01 & 2 \\
\hline & & & $f(x)=-2.193 \times x^{\wedge}(-21.76)+7.491$ & 0.28 & 0.46 & 1.11 & 3.70 & 3 \\
\hline \multirow[t]{4}{*}{ R Ventral } & \multirow[t]{2}{*}{ Incidental } & \multirow[t]{2}{*}{ Linear power } & $f(x)=0.3052+1.556$ & -0.29 & 0.03 & 3.11 & 29.05 & 3 \\
\hline & & & $f(x)=-3.491 \times x^{\wedge}(-24.6)+3.17$ & -0.35 & 0.33 & 3.18 & 20.23 & 2 \\
\hline & \multirow[t]{2}{*}{ Intentional } & \multirow[t]{2}{*}{ Linear power } & $f(x)=1.258 x+16.53$ & 0.38 & 0.53 & 2.15 & 13.90 & 3 \\
\hline & & & $f(x)=-5.599 \times x^{\wedge}(-21.48)+21.42$ & 0.69 & 0.84 & 1.52 & 4.64 & 2 \\
\hline
\end{tabular}

L, Left; Ventral, ventral stream; R, right; Linear, linear fit; Power, power fit; RMSE, root mean squared error; SSE, sum of squares error; DFE, degrees of freedom error.

(Table 3). In the incidental condition, neither the left nor the right ventral streams nor the left MTL was well fit by either function (all adjusted $R^{2}<0$ ). The right MTL was better fit by a power function with decreasing slope (adjusted $R^{2}=0.30$ and 0.05 for the power and linear fits, respectively). Note that, in each of these ROIs, the data point at $35^{\circ}$ rotation is significantly lower than the data point at both the $25^{\circ}$ and $55^{\circ}$ rotations. This point at $35^{\circ}$ does not predict the fit well, resulting in negative adjusted $R^{2}$ values for the left and right ventral streams and left MTL. In the intentional condition, the left ventral stream is well fit by a linear function (adjusted $R^{2}=0.98$ and 0.95 for the linear and power fits, respec- tively), whereas the right ventral stream is well fit by a power function with decreasing slope (adjusted $R^{2}=0.69$ and 0.38 for the power and linear fits, respectively). The left MTL was better fit by a power function with increasing slope (adjusted $R^{2}=0.42$ and 0.09 for the power and linear fits, respectively). The right MTL was better fit by a power function with decreasing slope (adjusted $R^{2}=0.28$ and 0.12 for the power and linear fits, respectively). This pattern (of flat responses in the ventral visual stream and power responses in the MTL) is consistent with a role of top-down processing modulating ventral stream activity that subsequently feeds into the hippocampus. 
A second set of ROIs was revealed by the intentional-incidental contrast that primarily consisted of default network regions. Activity in these regions appeared to be related to inattention in the (less engaging) incidental task and was not modulated by stimulus type (i.e., first, repeat, rotated). The coordinates of these ROIs are listed in Table 1 for archival purposes.

\section{Discussion}

Our principal investigation was to define the hippocampal pattern separation response function. Unique from previous studies, we explicitly controlled target-lure similarity using photographs of parametrically rotated objects. This allowed us to quantitatively define target-lure similarity and use stimulus change as an independent variable. Furthermore, by explicitly controlling stimulus similarity, we quantified the exact function of pattern separation and the hippocampal sensitivity to stimulus change, such that both high-similarity stimuli (i.e., objects rotated $15^{\circ}$ ) and low-similarity stimuli (i.e., objects rotated $55^{\circ}$ ) were treated like novel stimuli. Consistent with our predictions, we observed both left and right hippocampal pattern separation. Furthermore, task demands determined the laterality of the pattern separation.

In the incidental condition, pattern separation was observed in the left hippocampus, and activity was well fit by a power function with decreasing slope. In contrast, right hippocampal activity was better fit by a pattern completion linear function. In this condition, participants classified objects as toy or not a toy, relying on semantic information (i.e., task demands emphasized object identity more than object orientation). Left hippocampal activation is associated with verbal memory (Powell et al., 2007; Pereira et al., 2010). Left temporal lobectomy patients have impaired learning, recall, and recognition of prose passages (Milner, 1958; Frisk and Milner, 1990), word lists (Dennis et al., 1988; Baxendale, 1997), and object names (Incisa della Rocchetta and Milner, 1993). In normal controls, investigations using semantic classification of either animacy (i.e., "living" or "nonliving") (Otten and Rugg, 2001; Otten et al., 2001; Fliessbach et al., 2010) or concreteness (i.e., "concrete" or "abstract") (Baker et al., 2001) have led to stronger activation of the left hippocampus than nonsemantic discriminations. When classifying, participants use previous semantic knowledge of object identity to abstract its grouping under task constraints (Fliessbach et al., 2010). It is likely that left-lateralized pattern separation in the incidental task results from semantic inputs more strongly activating the left hippocampus.

In the intentional condition, pattern separation was observed in the right hippocampus, and activity was well fit by a power function with decreasing slope. In contrast, left hippocampal activity was better fit by a pattern completion power function with increasing slope. In this condition, participants identified objects as new, old, or rotated, relying on spatial information (i.e., task demands emphasized object orientation more than object identity). Right hippocampal activation is associated with navigational skills (Maguire et al., 1997; Iaria et al., 2008) and spatial memory (Köhler et al., 2005; Aradillas et al., 2011). Right temporal lobectomy patients have impaired spatial memory (Feigenbaum et al., 1996; Morris et al., 1996; Abrahams et al., 1997; Bohbot et al., 1998), topographical memory (Spiers et al., 2001), and memory dependent on the relationship between spatial cues and environmental reference (Finke et al., 2011). Spatial memory viewpoint dependence relies on mental rotation (compared with viewpoint-independent representations), as evidenced through response latencies for shifted-view images compared with control
Table 4. Mean RT for intentional task

\begin{tabular}{lll}
\hline Condition & Mean RT & $p$ value \\
\hline First & 1019.7 & \\
$0^{\circ}$ & 1340.0 & $4.4 e-11$ \\
$15^{\circ}$ & 1355.0 & $3.4 e-10$ \\
$25^{\circ}$ & 1354.9 & $2.9 e-10$ \\
$35^{\circ}$ & 1299.1 & $1.0 e-08$ \\
$55^{\circ}$ & 1285.1 & $7.2 e-08$ \\
\hline
\end{tabular}

A t test comparison between rotated conditions compared with first-presentation stimuli revealed significant differences between all rotated conditions and first-presentation stimuli. Within the rotated conditions, there was a linear trend for rotation, such that longer RTs were associated with smaller rotations. This pattern of response latency indicates mental rotation for rotated conditions.

images (King et al., 2002). Unfortunately, technical difficulties precluded accurately recording reaction times (RTs) in the fMRI task. We performed a behavioral follow-up experiment with the intentional task using 32 volunteers from the Brigham Young University community. When we examined the RT data, we observed a rotation-dependent response latency for all rotated conditions, with longer responses associated with smaller rotations (Table 4). This RT-rotation relationship is opposite that observed in classical mental rotation literature (Shepard and Metzler, 1971) because of task differences. In the classic visual comparison task, all stimuli are rotated and participants perform mental rotation to determine whether two simultaneously presented objects are the same image or mirror images. Participants in the intentional version of our task decide whether the object presented has been seen previously and, if so, whether it rotated from the original presentation. With large rotations, the difference is more obvious and RTs should be short. With small rotations, however, the difference should be less obvious and one predicts longer RTs for trials in which the object is correctly identified. Indeed, when we performed a one-way ANOVA on the RTs for rotated stimuli, we observed a main effect of rotation $\left(F_{(1,29)}=4.13, p=0.004\right)$ and, critically, a significant linear trend $\left(F_{(1,29)}=9.37, p=0.005\right)$. It is likely that right-lateralized pattern separation in the intentional task results from spatial inputs more strongly activating the right hippocampus.

Pattern separation of semantic information occurred in the left hippocampus in a semantic classification task and pattern separation of spatial information occurred in the right hippocampus in a mental rotation task. We conclude that pattern separation is the hippocampal default operation and is driven by the information most relevant to the task (Yassa and Stark, 2011). As the hippocampus receives a sufficient level of input (e.g., increased semantic input in the incidental task and increased spatial input in the intentional task), it easily pattern separates. It should be noted that, in other circumstances, the pattern of hippocampal activity may be reversed, as in the case when increased activity is observed in response to old items recognized with high confidence (Kirwan et al., 2009) or subjective recollection or source retrieval (Diana et al., 2007), presumably a consequence of pattern completion. In the current study, we hypothesized that the incidental task would allow isolation of hippocampal activity without the influence of top-down processes, whereas the intentional task would demonstrate the effects of task demands on pattern separation (Kirwan and Stark, 2007; Bakker et al., 2008). However, we see that left-lateralized pattern separation in our incidental task is influenced by semantic memory and thus also affected by top-down processes. Curves fit to the intentional data have better fits $\left(R^{2}=0.84\right.$ and 0.93 for the left and right hippocampus, respectively) than those fit to the incidental data $\left(R^{2}=\right.$ 0.62 and 0.33 for the left and right hippocampus, respectively), indi- 
cating that intentional memory encoding strongly biases the hippocampus toward pattern separation, but pattern separation still occurs in an incidental task.

We looked for an effect of task demand in the ventral stream inputs into the hippocampus. If task demands drive hippocampal pattern separation, this effect likely originates from ventral stream inputs and not in the hippocampus proper. If the effects of task demands originated in the hippocampus, this suggests that the hippocampus proper that would generate a different output for the same stimuli in different encoding conditions. The extrastriate cortex would merely relay the information about the perceived stimuli to the hippocampus, and thus activity in the extrastriate cortex would be identical for the same stimuli independent of the encoding condition. However, we see that activity in the ventral stream is different for the same stimuli depending on the encoding condition, suggesting that the effects of task demands is generated in the ventral stream and not in the hippocampus proper. Ventral stream activity mirrored hippocampal activity: pattern-separation-like curves were seen in the left hippocampus for incidental encoding and in the right hippocampus for intentional encoding, and pattern-completion-like curves were seen in the left hippocampus for intentional encoding and in the right hippocampus for incidental encoding. In contrast to hippocampal activity, ventral stream activity was not well fit by power or linear functions (Tables 2, 3), suggesting that top-down processing modulates information processed by the ventral stream but that ultimately it is the hippocampus that pattern separates by amplifying the signal received. Uncapher et al. (2011) demonstrated that attention alone is enough to alter perceptual stimuli processing. Our results are consistent with previous research showing that visual cortex generates a novelty signal (Danckert et al., 2007) and that top-down processing modifies inputs as they are received in visual cortex (Coutinho et al., 2010). Consistent with previous results (Reber et al., 1998), we observed decreased activation in the ventral stream during incidental encoding compared with intentional encoding. A previous study (Duncan et al., 2009) looking at hippocampal match-mismatch found that intentional encoding generated a match signal whereas incidental encoding generated a mismatch signal, suggesting that even in an incidental task the hippocampus is biased toward pattern separation. Studies looking at control mechanisms and how they interact with the MTL (Rugg et al., 1997; Golby et al., 2001; Uncapher et al., 2011; Minamoto et al., 2012) suggest both a frontal lobe-MTL interaction as well as a parietal lobe-MTL interaction. Additional research will be needed to better investigate the role and location of top-down processing and its interaction with MTL activity.

Our study was not designed to investigate task-dependent laterality effects, and thus the task demands of the incidental and intentional conditions are not entirely comparable. A $2 \times 2$ design intended to directly investigate pattern separation in spatial and verbal memory will better explore laterality in hippocampaldependent memory. Also, we were limited to a between-subjects design and thus were unable to directly examine the effect of task within subjects. Although it is possible to administer the incidental and then the intentional task to the same participant, it is not possible to do the reverse and keep the integrity of the incidental task design. Unable to counterbalance these two conditions, we elected a between-subjects design.

Last, when performing our second-level analysis on the hippocampus and MTL, we used the same old voxels as in our firstlevel new $>$ old contrast. The problem with twice-using voxels is that our new $>$ old contrast isolates voxels that have extremely low levels of activity for old stimuli, which could bias our secondlevel analysis toward finding pattern separation. One possible solution is to split the old stimuli, using half in the first-level analysis to define the ROI and the other half in the second-level curve-fitting analysis. When we performed this analysis, results were strikingly similar to our initial results, with ROIs of our split analysis overlapping the original analysis in 21 (of 58) voxels in the left hippocampus and 9 (of 10) voxels in the right hippocampus. Because splitting the data results in very few old trials in each category, we present here the analysis that uses all old trials to maximize power in the fMRI analyses.

We conclude that pattern separation is the hippocampal default operation as driven by information pertinent to the task. Top-down processes driven by task demands modulate inputs into the hippocampus via the ventral visual stream. Unique memory representations result from pattern-separation-like activity in the ventral stream that is subsequently amplified in the hippocampus.

\section{References}

Abrahams S, Pickering A, Polkey CE, Morris RG (1997) Spatial memory deficits in patients with unilateral damage to the right hippocampal formation. Neuropsychologia 35:11-24. CrossRef Medline

Amaral DG, Witter MP (1989) The three-dimensional organization of the hippocampal formation: a review of anatomical data. Neuroscience 31: 571-591. CrossRef Medline

Aradillas E, Libon DJ, Schwartzman RJ (2011) Acute loss of spatial navigational skills in a case of a right posterior hippocampus stroke. J Neurol Sci 308:144-146. CrossRef Medline

Avants BB, Epstein CL, Grossman M, Gee JC (2008) Symmetric diffeomorphic image registration with cross-correlation: evaluating automated labeling of elderly and neurodegenerative brain. Med Image Anal 12:2641. CrossRef Medline

Baker JT, Sanders AL, Maccotta L, Buckner RL (2001) Neural correlates of verbal memory encoding during semantic and structural processing tasks. Neuroreport 12:1251-1256. CrossRef Medline

Bakker A, Kirwan CB, Miller M, Stark CE (2008) Pattern separation in the human hippocampal CA3 and dentate gyrus. Science 319:1640-1642. CrossRef Medline

Baxendale SA (1997) The role of the hippocampus in recognition memory. Neuropsychologia 35:591-598. CrossRef Medline

Bohbot VD, Kalina M, Stepankova K, Spackova N, Petrides M, Nadel L (1998) Spatial memory deficits in patients with lesions to the right hippocampus and to the right parahippocampal cortex. Neuropsychologia 36:1217-1238. CrossRef Medline

Coutinho MV, Couchman JJ, Redford JS, Smith JD (2010) Refining the visual-cortical hypothesis in category learning. Brain Cogn 74:88-96. CrossRef Medline

Cox RW (1996) AFNI: software for analysis and visualization of functional magnetic resonance neuroimages. Comput Biomed Res 29:162-173. CrossRef Medline

Danckert SL, Gati JS, Menon RS, Köhler S (2007) Perirhinal and hippocampal contributions to visual recognition memory can be distinguished from those of occipito-temporal structures based on conscious awareness of prior occurrence. Hippocampus 17:1081-1092. CrossRef Medline

Daselaar SM, Fleck MS, Prince SE, Cabeza R (2006) The medial temporal lobe distinguishes old from new independently of consciousness. J Neurosci 26:5835-5839. CrossRef Medline

Dennis M, Farrell K, Hoffman HJ, Hendrick EB, Becker LE, Murphy EG (1988) Recognition memory of item, associative and serial-order information after temporal lobectomy for seizure disorder. Neuropsychologia 26:53-65. CrossRef Medline

Diana RA, Yonelinas AP, Ranganath C (2007) Imaging recollection and familiarity in the medial temporal lobe: a three-component model. Trends Cogn Sci 11:379-386. CrossRef Medline

Duncan K, Curtis C, Davachi L (2009) Distinct memory signatures in the hippocampus: Intentional states distinguish match and mismatch enhancement signals. J Neurosci 29:131-139. CrossRef Medline

Eichenbaum H, Yonelinas AP, Ranganath C (2007) The medial temporal 
lobe and recognition memory. Annu Rev Neurosci 30:123-152. CrossRef Medline

Feigenbaum JD, Polkey CE, Morris RG (1996) Deficits in spatial working memory after unilateral temporal lobectomy in man. Neuropsychologia 34:163-176. CrossRef Medline

Finke C, Ostendorf F, Braun M, Ploner CJ (2011) Impaired representation of geometric relationships in humans with damage to the hippocampal formation. PLoS One 6:e19507. CrossRef Medline

Fliessbach K, Buerger C, Trautner P, Elger CE, Weber B (2010) Differential effects of semantic processing on memory encoding. Hum Brain Mapp 31:1653-1664. CrossRef Medline

Forman SD, Cohen JD, Fitzgerald M, Eddy WF, Mintun MA, Noll DC (1995) Improved assessment of significant activation in functional magnetic resonance imaging (fMRI): use of a cluster-size threshold. Magn Reson Med 33:636-647. CrossRef Medline

Frisk V, Milner B (1990) The role of the left hippocampal region in the acquisition and retention of story content. Neuropsychologia 28:349359. CrossRef Medline

Geusebroek JM, Burghouts GJ, Smeulders AW (2005) The Amsterdam library of object images. Int J Comput Vision 6:103-112.

Golby AJ, Poldrack RA, Brewer JB, Spencer D, Desmond JE, Aron AP, Gabrieli JD (2001) Material-specific lateralization in the medial temporal lobe and prefrontal cortex during memory encoding. Brain 124:1841-1854. CrossRef Medline

Iaria G, Lanyon LJ, Fox CJ, Giaschi D, Barton JJ (2008) Navigational skills correlate with hippocampal fractional anisotropy in humans. Hippocampus 18:335-339. CrossRef Medline

Incisa della Rocchetta A, Milner B (1993) Strategic search and retrieval inhibition: the role of the frontal lobes. Neuropsychologia 31:503-524. CrossRef Medline

Kesner RP (2009) Tapestry of memory. Behav Neurosci 123:1-13. CrossRef Medline

King JA, Burgess N, Hartley T, Vargha-Khadem F, O'Keefe J (2002) Human hippocampus and viewpoint dependence in spatial memory. Hippocampus 12:811-820. CrossRef Medline

Kirwan CB, Stark CE (2007) Overcoming interference: an fMRI investigation of pattern separation in the medial temporal lobe. Learn Mem 14: 625-633. CrossRef Medline

Kirwan CB, Shrager Y, Squire LR (2009) Medial temporal lobe activity can distinguish between old and new stimuli independently of overt behavioral choice. Proc Natl Acad Sci U S A 106:14617-14621. CrossRef Medline

Klein A, Andersson J, Ardekani BA, Ashburner J, Avants B, Chiang MC, Christensen GE, Collins DL, Gee J, Hellier P, Song JH, Jenkinson M, Lepage C, Rueckert D, Thompson P, Vercauteren T, Woods RP, Mann JJ, Parsey RV (2009) Evaluation of 14 nonlinear deformation algorithms applied to human brain MRI registration. Neuroimage 46:786-802. CrossRef Medline

Köhler S, Danckert S, Gati JS, Menon RS (2005) Novelty responses to relational and non-relational information in the hippocampus and the parahippocampal region: a comparison based on event-related fMRI. Hippocampus 15:763-774. CrossRef Medline

Kubik S, Miyashita T, Guzowski JF (2007) Using immediate-early genes to map hippocampal subregional functions. Learn Mem 14:758-770. CrossRef Medline

Lacy JW, Yassa MA, Stark SM, Muftuler LT, Stark CE (2011) Distinct pattern separation related transfer functions in human CA3/dentate and CA1 revealed using high-resolution $\mathrm{fMRI}$ and variable mnemonic similarity. Learn Mem 18:15-18. CrossRef Medline

Lee I, Kesner RP (2004) Encoding versus retrieval of spatial memory: double dissociation between the dentate gyrus and the perforant path inputs into CA3 in the dorsal hippocampus. Hippocampus 14:66-76. CrossRef Medline

Leutgeb JK, Leutgeb S, Treves A, Meyer R, Barnes CA, McNaughton BL, Moser MB, Moser EI (2005) Progressive transformation of hippocampal neuronal representations in "morphed" environments. Neuron 48: 345-358. CrossRef Medline

Leutgeb S, Leutgeb JK (2007) Pattern separation, pattern completion, and new neuronal codes within a continuous CA3 map. Learn Mem 14:745757. CrossRef Medline

Leutgeb S, Leutgeb JK, Treves A, Moser MB, Moser EI (2004) Distinct en- semble codes in hippocampal areas CA3 and CA1. Science 305:12951298. CrossRef Medline

Maguire EA, Frackowiak RS, Frith CD (1997) Recalling routes around london: activation of the right hippocampus in taxi drivers. J Neurosci 17: 7103-7110. Medline

Marr D (1971) Simple memory: a theory for archicortex. Philos Trans R Soc Lond B Biol Sci 262:23-81. CrossRef Medline

McClelland JL, McNaughton BL, O'Reilly RC (1995) Why there are complementary learning systems in the hippocampus and neocortex: insights from the successes and failures of connectionist models of learning and memory. Psychol Rev 102:419-457. CrossRef Medline

Milner B (1958) Psychological defects produced by temporal lobe excision. Res Publ Assoc Res Nerv Ment Dis 36:244-257. Medline

Minamoto T, Osaka M, Engle RW, Osaka N (2012) Incidental encoding of goal irrelevant information is associated with insufficient engagement of the dorsal frontal cortex and the inferior parietal cortex. Brain Res 1429: 82-97. CrossRef Medline

Morris RG, Pickering A, Abrahams S, Feigenbaum JD (1996) Space and the hippocampal formation in humans. Brain Res Bull 40:487-490. CrossRef Medline

Norman KA, O'Reilly RC (2003) Modeling hippocampal and neocortical contributions to recognition memory: a complementary-learningsystems approach. Psychol Rev 110:611-646. CrossRef Medline

O’Reilly RC, Rudy JW (2001) Conjunctive representations in learning and memory: principles of cortical and hippocampal function. Psychol Rev 108:311-345. CrossRef Medline

Otten LJ, Rugg MD (2001) Task-dependency of the neural correlates of episodic encoding as measured by fMRI. Cereb Cortex 11:1150-1160. CrossRef Medline

Otten LJ, Henson RN, Rugg MD (2001) Depth of processing effects on neural correlates of memory encoding: relationship between findings from across- and within-task comparisons. Brain 124:399-412. CrossRef Medline

Pereira FR, Alessio A, Sercheli MS, Pedro T, Bilevicius E, Rondina JM, Ozelo HF, Castellano G, Covolan RJ, Damasceno BP, Cendes F (2010) Asymmetrical hippocampal connectivity in mesial temporal lobe epilepsy: evidence from resting state fMRI. BMC Neurosci 11:66. CrossRef Medline

Powell HW, Richardson MP, Symms MR, Boulby PA, Thompson PJ, Duncan JS, Koepp MJ (2007) Reorganization of verbal and nonverbal memory in temporal lobe epilepsy due to unilateral hippocampal sclerosis. Epilepsia 48:1512-1525. CrossRef Medline

Reber PJ, Stark CE, Squire LR (1998) Cortical areas supporting category learning identified using functional MRI. Proc Natl Acad Sci U S A 95: 747-750. CrossRef Medline

Rotshtein P, Henson RN, Treves A, Driver J, Dolan RJ (2005) Morphing Marilyn into Maggie dissociates physical and identity face representations in the brain. Nat Neurosci 8:107-113. CrossRef Medline

Rugg MD, Fletcher PC, Frith CD, Frackowiak RS, Dolan RJ (1997) Brain regions supporting intentional and incidental memory: a PET study. Neuroreport 8:1283-1287. CrossRef Medline

Shepard RN, Metzler J (1971) Mental rotation of three-dimensional objects. Science 171:701-703. CrossRef Medline

Spiers HJ, Burgess N, Maguire EA, Baxendale SA, Hartley T, Thompson PJ, O'Keefe J (2001) Unilateral temporal lobectomy patients show lateralized topographical and episodic memory deficits in a virtual town. Brain 124:2476-2489. CrossRef Medline

Squire LR, Stark CE, Clark RE (2004) The medial temporal lobe. Annu Rev Neurosci 27:279-306. CrossRef Medline

Talairach J, Tournoux P (1988) A co-planar stereotaxic atlas of the human brain. New York: Thieme Medical.

Treves A, Rolls ET (1994) Computational analysis of the role of the hippocampus in memory. Hippocampus 4:374-391. CrossRef Medline

Uncapher MR, Hutchinson JB, Wagner AD (2011) Dissociable effects of top-down and bottom-up attention during episodic encoding. J Neurosci 31:12613-12628. CrossRef Medline

Vazdarjanova A, Guzowski JF (2004) Differences in hippocampal neuronal population responses to modifications of an environmental context: evidence for distinct, yet complementary, functions of CA3 and CA1 ensembles. J Neurosci 24:6489-6496. CrossRef Medline

Wills TJ, Lever C, Cacucci F, Burgess N, O’Keefe J (2005) Attractor dynamics in the hippocampal representation of the local environment. Science 308:873-876. CrossRef Medline 
Xiong J, Gao JH, Lancaster JL, Fox PT (1995) Clustered pixel analysis for functional MRI activation studies of the human brain. Hum Brain Mapp 3:287-301. CrossRef

Yassa MA, Stark CE (2011) Pattern separation in the hippocampus. Trends Neurosci 34:515-525. CrossRef Medline
Yassa MA, Stark SM, Bakker A, Albert MS, Gallagher M, Stark CE (2010) High-resolution structural and functional MRI of hippocampal CA3 and dentate gyrus in patients with amnestic mild cognitive impairment. Neuroimage 51:1242-1252. CrossRef Medline 